Z. klin. Chem. u. klin. Biochem.

9. Jg., S. 314-319, Juli 1971

\title{
Tierexperimentelle Untersuchungen zum Protein- und Aminosäurestoffwechsel nach standardisierten Verbrennungen
}

\author{
Von H. KRöNER, J. BeIEN und W. StAIB \\ Institut fïr Pbysiologische Chemie der Universität Düsseldorf
}

,

(Eingegangen am 7. Dezember 1970)

\begin{abstract}
Es wird der Einfluß einer standardisierten Verbrennung auf den Aminosäure- und Proteinstoffwechsel der Ratte untersucht. Die Gesamtaminosäuren des Plasmas zeigen einen zweigipfligen Anstieg, während sich die Spiegel der einzelnen Aminosäuren sehr unterschiedlich verhalten. Gemessen am Einbau von Leucin- $\left[1{ }^{14} \mathrm{C}\right]$ ist die Synthese des Leberproteins, nicht aber die der in der Leber gebildeten Plasmaproteine gehemmt. Gleichzeitig ist der Leucinabbau gemessen als ${ }^{14} \mathrm{CO}_{2}$-Bildung beim verbrennungskranken Tier stark gesteigert. Dieser Befund ist nur am Ganztier, nicht aber an der isoliert perfundierten Leber zu erheben, während sich die erwähnte Störung der Proteinsynthese auch dort manifestiert. Die Befunde sprechen für verschiedene Störungen des Protein- und Aminosäurestoffwechsels nach Verbrennungen, die Ursachen und Zusammenhänge werden diskutiert.
\end{abstract}

\section{The effect of standardised burning on the protein and amino acid metabolism of animals}

Studies are reported on the effects of standardised burning on the amino acid and protein metabolism of the rat. After the animal had been burned, the total amino acids of the plasma showed an increase with two maxima, but there were wide differences in the behaviour of the individual amino acids. Measured as the incorporation of $\left[1-{ }^{14} \mathrm{C}\right]$ leucine, the synthesis of plasma proteins in the liver was unaffected, but the synthesis of liver proteins was inhibited. At the same time, the degradation of leucine, measured as the formation of ${ }^{14} \mathrm{CO}_{2}$, was markedly increased. The disturbance of protein synthesis can be reproduced in isolated, perfused liver, but the increased degradation of leucine is only observed in whole animals. These findings indicate that burning influences various stages in the metabolism of proteins and amino acids. The causes and interrelationships of these affects are discussed.

In einer früheren Untersuchung (1) fanden wir außer einem Anstieg der freien Aminosäuren des Serums in der Schockphase auch einen Anstieg derselben in Serum und Leber während der späten Phase der Verbrennungskrankheit. $\mathrm{Da}$ zu diesem späten Zeitpunkt auch der Energiequotient ATP/ADP in der Leber erniedrigt gefunden wurde, haben wir zur Erklärung damals eine Störung der Proteinsynthese in der Leber angenommen. Von den verschiedenen metabolischen Veränderungen (2) nach Verbrennungen dürfte die Störung des Proteinstoffwechsels besonders schwerwiegend sein. Wir haben daher versucht, diese Frage weiter abzuklären. Aufschluß über die Art der Stoffwechselstörung erwarteten wir von einer Analyse der einzelnen Aminosäuren im Serum. Unterschiedliche Einwirkung auf den Aminosäurestoffwechsel z. B. in der Schockphase und in der Spätphase sollten sich auf die Spiegel der einzelnen Aminosäuren im Serum unterschiedlich auswirken. Untersuchungen über den Verbleib einer einzelnen ${ }^{14} \mathrm{C}$-markierten Aminosäure sollten erkennen lassen, ob unsere Annahme einer Proteinsynthesestörung richtig war. Entsprechende Versuche an der isoliert perfundierten Leber müßten ergeben, ob die Störungen des Aminosäurestoffwechsels Folge einer manifesten Veränderung in der Leber oder aber Folge von Auswirkungen der Körperperipherie sind. Letztere dürften sich in der isoliert perfundierten Leber nicht bemerkbar machen.

\section{Methodik}

Die als Versuchstiere benutzten männlichen Wistarratten (220 bis $300 \mathrm{~g}$, Brünger, Bokel) erhielten Standardkost (Höveler, Langen- feld) und Wasser ad libitum. Etwa 24 Stdn. vor Blutentnahme, Perfusion bzw. Abatmungsversuch wurde ihnen das Futter entzogen, das Wasser belassen,

Den Tieren wurden mit der früher angegebenen Methodik (3) - 10 Sek., $250^{\circ}, 25 \%$ det Oberfläche - Verbrennungen gesetzt. Wir verglichen in den nachfolgenden Untersuchungen Normaltiere mit Tieren, denen 4 Stdn., 6, 10 und 15 Tage vorher Verbrennungen gesetzt wurden. Besondere Vorkehrungen, wie Infektionsprophylaxe und Kompensation des Flüssigkeits- und Elektrolytverlustes, wurden nicht getroffen.

Für die Aminosäureanalyse benötigten wir $10 \mathrm{ml}$ Plasma. Dazu wurde von $2-3$ Ratten durch Aortenpunktion in Äthernarkose Blut gewonnen und mit 1-2 Tropfen Liquemin (HoffmannLa Roche) versetzt, gemischt und $10 \mathrm{Min}$. bei $3000 \mathrm{U} / \mathrm{Min}$. zentrifugiert. Das Plasma wurde 1:5 mit 1 proz. Pikrinsäure (4) enteiweißt der Überstand an einer Dowex $2 \times 10$ Säule von Pikrinsäure befreit und das Eluat im Rotationsverdampfer auf etwa $1 \mathrm{ml}$ eingeengt. Den $\mathrm{pH}$ stellten wir mit $1 \mathrm{~N} \mathrm{NaOH}$ auf 7-8 ein. Nach 4 Stdn. wurde der $\mathrm{pH}$ mit $1 \mathrm{~N} \mathrm{HCl}$ auf 2,2 gesenkt und die Lösung mit $\mathrm{H}_{2} \mathrm{O}$ auf $5 \mathrm{ml}$ aufgefüllt. Die Probe wurde bis zur Analyse eingefroren aufbewahrt. Zur Analyse benutzten wir einen von ZIMMERMANN-TELSCHOW (5) beschriebenen, leicht modifizierten automatischen Aminosäureanalysator. Die geringen Verluste an der Dowex $2 \times 10$ Säule wurden für die einzelnen Aminosäuren ermittelt, die Meßwerte entsprechend korrigiert.

In einer anderen Versuchsteihe wurde der Leucinstoffwechsel in Abhängigkeit von der Verbrennung untersucht. Wir bestimmten ${ }^{14} \mathrm{CO}_{2}$-Abatmung und ${ }^{14} \mathrm{C}$-Einbau in Leber- und Plasmaprotein nach intraperitonealer Injektion von $1 \mu \mathrm{C}$ Leucin-[1-14C $\mathrm{C}-$ Lösung/ $100 \mathrm{~g}$ Körpergewicht $(50 \mu \mathrm{C}$ Leucin-[1-14 C] $/ 10 \mathrm{ml} \quad 0,9$ proz. $\mathrm{NaCl}-\mathrm{Lösung}$ ). Aus einem Stoffwechselkäfig (6) leiteten wir die Ausatmungsluft durch ein $\mathrm{CO}_{2}$-Absorptionsgemisch (7) (Äthanolamin: Methylcellosolve $=1: 2 \quad \nabla / v)$. Die Absorptionsgemischröhrchen wurden $30,60,90,120,180,240$ und $300 \mathrm{Min}$. nach der Injektion gewechselt, die Proben unter Zusatz von Diotol (8) im Flüssigkeits-Scintillations-Zähler gezählt. Wir berechneten den Prozentsatz der ${ }^{14} \mathrm{C}$-Ausgangsdosis, der als ${ }^{14} \mathrm{CO}_{2}$ abgeatmet wurde. Nach den 5 Stdn. Versuchsdauer im Stoffwechselkäfig 
wurde wie oben beschrieben Plasma gewonnen und die Lebern entnommen. Etwa $0,5 \mathrm{~g}$ Leber wurde $2 \mathrm{Min}$. unter Eiskühlung mit Wasser im Verhältnis 1:5 (w/w) homogenisiert. Zu $0,5 \mathrm{ml}$ Plasma bzw. $1 \mathrm{ml}$ Leberhomogenat gaben wir jeweils $1 \mathrm{ml} 10$ proz. Trichloressigsäure $(0,2 \mathrm{~N}$ Leucin als Träger) zentrifugierten $15 \mathrm{Min}$. bei $3000 \mathrm{U} / \mathrm{Min}$. und wuschen den Rückstand mit $5 \mathrm{ml}$ 5 proz. Trichloressigsäure $(0,2 \mathrm{~N}$ an Leucin als Träger) nach. Der Rückstand wurde in $0,5 \mathrm{ml} \mathrm{H}_{2} \mathrm{O}$ aufgeschwemmt und unter Zugabe von $2 \mathrm{ml} 1 \mathrm{~N} \mathrm{NaOH}$ gelöst. Von dieser alkalischen Proteinlösung nahmen wir $0,5 \mathrm{ml}$ zur Proteinbestimmung nach der Biuretmethode (9). Zur Messung der Radioaktivität im Flüssigkeitsscitillationszähler wurden $0,5 \mathrm{ml}$ mit $1,5 \mathrm{ml}$ Hyamine und $10 \mathrm{ml}$ Diotol versetzt. Mit Hilfe des externen Standards wurde der Quench korrigiert.

Der Leucinstoffwechsel wurde auch an isoliert perfundierten Lebern untersucht. Die Lebern verbrennungskranker und normaler Tiere wurden präpariert $(10,11)$ und mit dem halbsynthetischen Medium von Schimassek (12) in einer von MILLER (13) beschriebenen Apparatur perfundiert. Dem Perfusionsmedium wurde ein Aminosäuregemisch nach Exton und PARK (14) zugesetzt. Nach 30 Min. Perfusion gaben wir $2 \mu \mathrm{C}$ Leucin-[1_14 C] (in $1 \mathrm{ml} 0,9$ proz. $\mathrm{NaCl}$ ) hinzu. $30 \mathrm{Min}$. später entnahmen wir $2 \mathrm{ml}$ Blut und ein Leberläppchen, ebenso nach 60 und $120 \mathrm{Min}$. Das während der Perfusion gebildete ${ }^{14} \mathrm{CO}_{2}$ wurde mittels einer Wasserstrahlpumpe abgesaugt und wie oben beschrieben absorbiert und gemessen. Plasma- und Leberprotein und die eingebaute ${ }^{14} \mathrm{C}$-Aktivität bestimmten wir ebenfalls nach der oben erwähnten Methode.

Die Angaben der ${ }^{14} \mathrm{C}$-Aktivität wurde bezogen auf $\mathrm{g}$ Leber, da hier die ${ }^{14} \mathrm{C}$-Dosis einheitlich war, während bei den Ganztierversuchen die Dosierung individuell pro $\mathrm{kg}$ Körpergewicht erfolgte.

Die statistische Auswertung der Ergebnisse erfolgte nach dem t-Test.

\section{Ergebnisse}

Einfluß von Verbrennungen auf den Aminosäurengehalt des Blutes

Mit unserer Aminosäurenanalyse ließen sich folgende Aminosäuren bestimmen (in Klammern der Normalwert in $\mathrm{mg} / \mathrm{l}$ Plasma): Taurin (27,4), Asparaginsäure $(2,16)$, Threonin $(24,1)$, Serin $(39,2)$, Glutaminsäure $(13,4)$, Prolin $(17,3)$, Glycin $(23,8)$, Alanin $(20,1)$, Cystein $(3,0)$, Valin $(18,1)$, Methionin (5,7), Isoleucin $(9,9)$, Leucin $(17,6)$, Tyrosin $(10,8)$, Phenylalanin $(8,2)$, Ornithin (10,0), Lysin (33,1); Histidin, Tryptophan und Arginin konnten wir wegen zu geringer Konzentration nicht quantitativ bestimmen. Dagegen ließen sich mit unserer Methode Harnstoff $(20,7)$ und Ammoniak $(35,1)$ erfassen (vgl. Tab. 1).

Der Gesamtaminosäurenspiegel, einschließlich Harnstoff und Ammoniak, betrug bei den Kontrolltieren $339,8 \mathrm{mg} / \mathrm{l}$ Plasma.

Bereits 4 Stdn. nach der Verbrennung ist die Summe der Aminosäuren im Plasma um 39\% signifikant auf $474 \mathrm{mg} / \mathrm{l} \mathrm{Plasma}$ angestiegen.

Der 6-Tagewert, $380,7 \mathrm{mg} / \mathrm{l}$ Plasma und der 10-Tagewert, $424,3 \mathrm{mg} / \mathrm{l}$ Plasma waren gegenüber dem Kontrollwert nicht signifikant erhöht. Dagegen lag der 15-Tagewert mit $476,5 \mathrm{mg} / \mathrm{l}$ Plasma um $40 \%$ signifikant über dem der Kontrollen. Diese Befunde bestätigen frühere Gesamt-Aminosäurenbestimmungen an verbrannten Tieren (1).

Tab. 1

Aminosäurengehalt in $\mathrm{mg} / \mathrm{l}(\overline{\mathrm{x}} \pm \mathrm{s})$ Plasma bei Kontrollen, Tieren 4 Stdn., 6, $10 \mathrm{u} .15$ Tage nach Verbrennung (in Klammern die prozentuale Abweichung vom Normaltier bei den signifikant erhöhten $(+)$ bzw. verminderten $(-)$ Werten) $p<0,05$

\begin{tabular}{|c|c|c|c|c|c|c|}
\hline \multirow[b]{2}{*}{ Taurin } & \multicolumn{2}{|c|}{$\begin{array}{l}n=10 \\
\text { Normal }\end{array}$} & \multirow{2}{*}{$\frac{\begin{array}{c}n=5 \\
4 \text { Stunden }\end{array}}{\begin{array}{l}83,5 \pm 16,1 \\
(+204 \%)\end{array}}$} & $\begin{array}{r}n=4 \\
6 \text { Tage }\end{array}$ & $\begin{array}{r}n=5 \\
10 \text { Tage }\end{array}$ & $\begin{array}{c}n=7 \\
15 \text { Tage }\end{array}$ \\
\hline & 27,4 & $\pm 5,8$ & & $31,2 \pm 0,6$ & $32,0 \pm$ & $33,7 \pm 12,0$ \\
\hline Harnstoff & 20,7 & $\pm 4,7$ & $\begin{array}{l}45,4 \pm \frac{8,7}{19 \%)} \\
(+119 \%\end{array}$ & $26,9 \pm 5,8$ & $23,0 \pm$ & ${ }_{(+55 \%)}^{32,1} \stackrel{8,2}{ }$ \\
\hline Asparaginsäure & 2,16 & $\pm 0,6$ & $\begin{array}{l}7,1 \pm 7,0 \\
(+228 \%)\end{array}$ & $1,7 \pm 0,3$ & $2,5 \pm$ & $3,56 \pm 2,1$ \\
\hline Threonin & 24,1 & $\pm 5,6$ & $39,1 \pm 24,1$ & $17,6 \pm 3,6$ & ${ }_{\left(-\frac{1}{30} \%\right)}^{0,9}$ & $\begin{array}{c}44,9 \pm \underset{ }{(+85 \%)} \\
4,6\end{array}$ \\
\hline Serin & 39,2 & $\pm \quad 4,6$ & $30,2 \pm 11,5$ & ${ }_{(-26 \%)}^{2,6} \underset{\%}{2,9}$ & ${ }_{(-42 \%)}^{1,9}$ & $39,1 \pm 12,8$ \\
\hline Glutaminsäure & 13,4 & $\pm 5,2$ & $14,4 \pm 6,5$ & $15,4 \pm 1,7$ & $13,0 \pm 2,7$ & 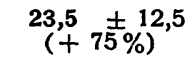 \\
\hline Prolin & 17,3 & $\pm 3,8$ & $21,7 \pm 8,6$ & $19,8 \pm 4,2$ & $22,6 \pm$ & $\begin{array}{c}36,5 \pm 10,0 \\
(+111 \%)\end{array}$ \\
\hline Glycin & 23,8 & $\pm 4,8$ & $22,4 \pm 5,0$ & ${ }_{(-47 \%)}^{12,5} \pm$ & $\left(-\frac{7,5}{6} \%\right)^{1,5}$ & $23,6 \pm 6,8$ \\
\hline Alanin & 20,1 & $\pm \quad 4,1$ & $6,7 \pm 6,6$ & ${ }_{(-29 \%)}^{14,2}$ & $15,6 \pm 5,2$ & $c_{(+29 \%)}^{25,9}$ \\
\hline Valin & 18,1 & $\pm \quad 0,6$ & $\left.{ }^{27,7} \underset{+5}{ \pm} \%\right)^{3,5}$ & $\begin{array}{c}30,5 \pm 12,3 \\
(+69 \%)\end{array}$ & $23,0 \pm 8,1$ & ${ }_{(+48 \%)}^{26,9} \underset{ }{ \pm, 1}$ \\
\hline Methionin & 5,7 & $\pm 0,6$ & $\begin{array}{l}3,8 \\
(-33 \%)\end{array}$ & $\begin{array}{l}3,0 \div 0,8 \\
(-47 \%)\end{array}$ & ${ }_{(-56 \%)}^{2,6} 1,0$ & $6,5 \pm 1,2$ \\
\hline Isoleucin & 9,9 & $\pm \quad 1,7$ & $11,2 \pm 2,9$ & $\begin{array}{c}14,0 \pm 2,1 \\
(+41 \%)\end{array}$ & $12,0 \pm 4,8$ & $\left.{ }^{12,7}+28 \%\right)^{2,7}$ \\
\hline Leucin & 17,6 & $\pm 4,4$ & $21,1 \pm 6,6$ & $\begin{array}{c}27,0 \pm 5,6 \\
(+53 \%)\end{array}$ & $22,8 \pm$ & $\begin{array}{c}25,6 \\
(+45 \%)\end{array}$ \\
\hline Tyrosin & 10,8 & $\pm 2,0$ & $11,7 \pm 3,6$ & $12,4 \pm 2,4$ & $\begin{array}{c}\left.15,1+\frac{1}{40} \%\right) \\
(+, 5\end{array}$ & $12,0 \pm 2,4$ \\
\hline Phenylalanin & 8,2 & $\pm 1,6$ & $9,7 \pm 3,6$ & $\begin{array}{c}10,8 \pm 1,9 \\
(+32 \%)\end{array}$ & $\begin{array}{c}12,9 \pm \frac{ \pm}{(+57 \%)} \\
(+5,6\end{array}$ & 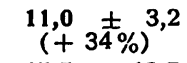 \\
\hline Ornithin & 10,0 & $\pm 7,0$ & $13,7 \pm 11,2$ & $17,0 \pm 7,4$ & $8,7 \pm 2,9$ & $15,5 \pm 13,7$ \\
\hline Lysin & 33,1 & $\pm 13,1$ & $48,0 \pm 16,5$ & $42,2 \pm 8,0$ & $\begin{array}{c}59,4 \pm 16,1 \\
(+80 \%)\end{array}$ & $49,7 \pm 26,2$ \\
\hline $\mathrm{NH}_{3}$ & 35,1 & $\pm 8,5$ & $39,0 \pm 7,5$ & $41,2 \pm 10,1$ & $34,3 \pm 12,2$ & $33,3 \pm 5,6$ \\
\hline Summe & 339,8 & $\pm 37,1$ & $\begin{array}{c}474,2 \pm 93,3 \\
(+39 \%)\end{array}$ & $380,7 \pm 49,6$ & $424,3 \pm 127,2$ & $\begin{array}{c}476,5 \pm 82,9 \\
(+40 \%)\end{array}$ \\
\hline
\end{tabular}


Aus der fraktionierten Aminosäurenanalyse ging hervor, daß bereits 4 Stdn. nach Verbrennung Taurin ( $+204 \%$ ), Harnstoff $(+119 \%)$, Asparaginsäure $(+228 \%)$ und Valin $(+53 \%)$ signifikant angestiegen und Methionin $(-33 \%)$ signifikant abgefallen waren. Die übrigen Aminosäuren lagen im normalen Streubereich. 6 Tage nach der Verbrennung hatten wir ein völlig anderes Bild. Diesmal fanden wir bei Valin $(+69 \%)$, Isoleucin $(+41 \%)$, Leucin $(+53 \%)$ und Phenylalanin $(+32 \%)$ eine signifikante Erhöhung, dagegen bei Serin $(-26 \%)$, Glycin $(-47 \%)$, Alanin $(-29 \%)$ und Methionin $(-47 \%)$ eine signifikante Verminderung gegenüber den Kontrolltieren.

Am 10. Tage fielen Threonin (-30\%), Serin $(-42 \%)$, Glycin (-68\%) und Methionin (-56\%) signifikant $\mathrm{ab}$, dagegen fand sich bei Tyrosin $(+40 \%)$, Phenylalanin $(+57 \%)$ und Lysin $(+80 \%)$ ein signifikanter Anstieg. Am 15. Tage fanden sich signifikante Erhöhungen einer großen Anzahl von Aminosäuren: Harnstoff $(+55 \%)$, Threonin $(+85 \%)$, Glutaminsäure $(+75 \%)$, Prolin $(+11 \%)$, Alanin $(+29 \%)$, Valin $(+48 \%)$, Isoleucin $(+28 \%)$, Leucin $(+45 \%)$ und Phenylalanin $(+34 \%)$. Die nicht erwähnten Aminosäuren lagen im Streubereich der Kontrolltiere.

Einfluß von Verbrennungen auf den Leucinstoffwechsel der lebenden Ratte

$\mathrm{Zu}$ verschiedenen Zeiten nach Leucin-[1-14C]-Injektion bestimmten wir, wieviel Prozent der ${ }^{14} \mathrm{C}$-Dosis als ${ }^{14} \mathrm{CO}_{2}$ abgeatmet wurde. Dabei berechneten wir die Werte einmal als Prozentsatz der Dosis, der pro Stunde abgeatmet wurde (Tab. 2), zum anderen wurde die Summe der einzelnen Werte in Prozent der Dosis

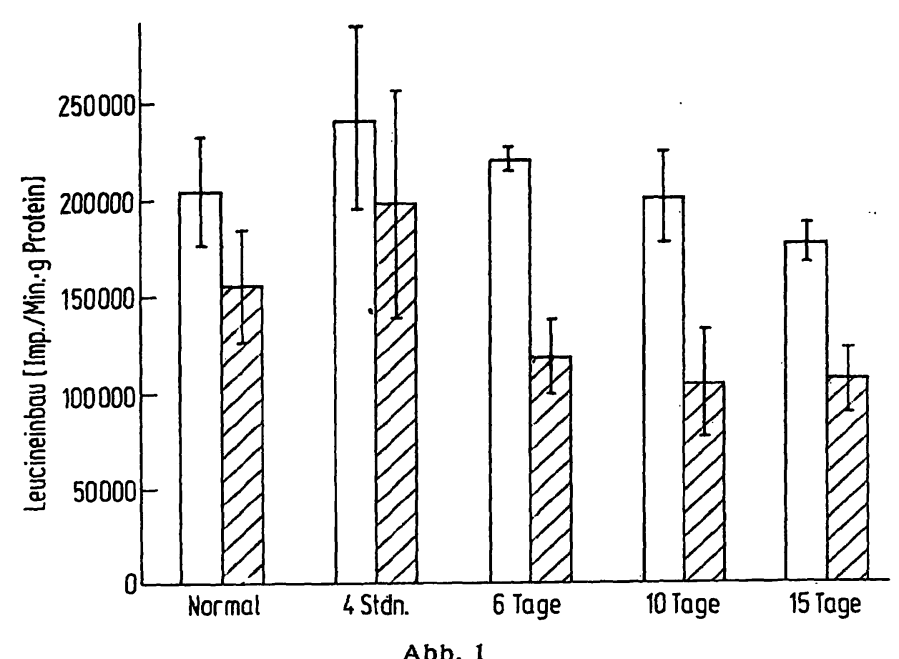

Einbau von Leucin-[1-1+C] in das Leberprotein (schraffierte Säulen) und die Plasmaproteine (weiße Säulen) normaler und verbrannter Ratten. $1 \mu \mathrm{C}$ Leucin-[1-14 $\mathrm{C}] / 100 \mathrm{~g}$ Körpergewicht wurde $5 \mathrm{Stdn}$. vor der Tötung der Tiere i. p. injiziert. Auf der Abszisse ist die Zeit nach der Verbrennung angegeben. Eingezeichnet sind Mittelw
destens 5 Tieren \pm Standardabweichung

kumulativ (Tab. 3) angegeben. Der Einbau ins Leberund Plasmaprotein wurde nach $5 \mathrm{Stdn}$. als Imp./Min. pro g Protein erfaßt. (Abb.1)

Bei den Kontrolltieren wurde in der ersten halben Stunde der größte Prozentsatz an ${ }^{14} \mathrm{CO}_{2}$ freigesetzt. Er nahm dann laufend ab. Zwei Stunden nach der Injektion war bereits kaum noch Aktivität zu messen (4,79\% gegenüber $24,98 \%$ in der ersten halben Stunde). In das Plasmaprotein wurde von den Kontrollen mit 205481 Imp./Min. ·g Protein deutlich mehr Leucin ${ }^{14} \mathrm{C}$ eingebaut als in das Leberprotein mit $156053 \mathrm{Imp} . /$ Min. - g Protein. 4 Stdn. nach der Verbrennung konnten weder bei der ${ }^{14} \mathrm{CO}_{2}$-Abspaltung noch beim fünf-

Tab. 2

$1 \mathrm{CO}_{2}$-Abatmung in $\%$ der Dosis/Stunde $(\overline{\mathrm{x}} \pm \mathrm{s})$ nach intraperitonealer Injektion von Leucin-[1-1 $\left.\mathrm{C}\right](1 \mu \mathrm{C} / 100 \mathrm{~g} \mathrm{Körpergewicht).} \mathrm{Die} \mathrm{Tiere} \mathrm{erhielten}$ 4 Stdn., 6, 10 oder 15 Tage vor der Leucininjektion eine Verbrennung, Kontrolltiere waren unbehandelt. $* \mathrm{p}<0,05 \quad * * \mathrm{p}<0,01 \quad * * * \mathrm{p}<0,001$

\begin{tabular}{|c|c|c|c|c|c|c|}
\hline $\begin{array}{l}\text { Zeit nach Leu- } \\
\text { cin-Injektion }\end{array}$ & $\begin{array}{l}\text { Kontrollen } \\
n=6\end{array}$ & $\begin{array}{l}4 \text { Stunden } \\
n=5\end{array}$ & & $\begin{array}{l}6 \text { Tage } \\
n=5\end{array}$ & $\begin{array}{c}10 \text { Tage } \\
n=5\end{array}$ & $\begin{array}{c}15 \text { Tage } \\
n=5\end{array}$ \\
\hline $0-30 \mathrm{Min}$. & $24,98 \pm 3,8$ & $22,50 \pm 1,64$ & 45,39 & $\pm 13,05 * *$ & $44,65 \pm 15,02 * * *$ & $46,83 \pm 5,03^{* * *}$ \\
\hline $30-60 \mathrm{Min}$. & $18,71 \pm 2,69$ & $18,34 \pm 2,24$ & 27,9 & $\pm 1,17 * * *$ & $20,09 \pm 3,1$ & $39,23 \pm 6,6 * * *$ \\
\hline $60-90 \mathrm{Min}$. & $11,29 \pm 0,78$ & $12,58 \pm 2,02$ & 14,07 & $\pm 1,88 * *$ & $15,22 \pm 2,03 * * *$ & $15,94 \pm 3,41 * *$ \\
\hline $90-120 \mathrm{Min}$. & $4,79 \pm 0,56$ & $4,70 \pm 0,88$ & 6,96 & $\pm 0,53 * * *$ & $8,60 \pm 1,64 * * *$ & $8,93 \pm 4,74$ \\
\hline $120-180 \mathrm{Min}$. & $2,36 \pm 0,33$ & $4,34 \pm 0,48$ & 3,58 & $\pm \quad 0,49 * * *$ & $3,03 \pm 0,64$ & $3,21 \pm 1,82$ \\
\hline $180-240 \mathrm{Min}$. & $1,15 \pm 0,19$ & $1,13 \pm 0,21$ & 1,91 & $\pm 0,13 * *$ & $1,74 \pm 0,23^{* *}$ & $1,63 \pm 0,74$ \\
\hline $240-300 \mathrm{Min}$. & $0,93 \pm 0,15$ & $0,85 \pm 0,23$ & 1,38 & $\pm 0,05 * * *$ & $1,11 \pm 0,17$ & $1,33 \pm 0,52$ \\
\hline
\end{tabular}

Tab. 3

${ }^{1}{ }^{4} \mathrm{CO}_{2}$-Abatmung in $\%$ der Dosis kumulativ $(\bar{x} \pm s)$ nach intraperitonealer Injektion von Leucin-[1-1، $\left.\mathrm{C}\right](1 \mu \mathrm{C} / 100 \mathrm{~g}$ Körpergewicht). Die Tiere erhielten 4 Stdn., 6, 10 oder 15 Tage vor der Leucininjektion eine Verbrennung, Kontrolltiere waren unbehandelt. $* \mathrm{p}<0,05 \quad * * \mathrm{p}<0,01 \quad * * * \mathrm{p}<0,001$

\begin{tabular}{|c|c|c|c|c|c|}
\hline $\begin{array}{l}\text { Zeit nach Leu- } \\
\text { cin-Injektion }\end{array}$ & $\begin{array}{l}\text { Kontrollen } \\
n=6\end{array}$ & $\begin{array}{c}4 \text { Stunden } \\
n=5\end{array}$ & $\begin{array}{l}6 \text { Tage } \\
n=5\end{array}$ & $\begin{array}{c}10 \text { Tage } \\
n=5\end{array}$ & $\begin{array}{c}15 \text { Tage } \\
\mathrm{n}=5\end{array}$ \\
\hline $0-30 \mathrm{Min}$. & $12,49 \pm 1,9$ & $11,25 \pm 0,82$ & $22,68 \pm 6,54 * *$ & $22,34 \pm 7,47 *$ & $23,41 \pm 2,51 * * *$ \\
\hline $30-60$ Min. & $21,84 \pm 3,24$ & $20,42 \pm 1,93$ & $36,64 \pm 7,1 * *$ & $37,39 \pm 5,99 * * *$ & $43,02 \pm 4,25 * * *$ \\
\hline $60-90$ Min. & $27,49 \pm 3,55$ & $26,71 \pm 2,76$ & $44,68 \pm 8,05^{* *}$ & $45,08 \pm 5,86^{* * *}$ & $51,00 \pm 3,41 * * *$ \\
\hline $90-120 \mathrm{Min}$. & $29,85 \pm 3,45$ & $29,31 \pm 2,70$ & $48,16 \pm 8,29 * * *$ & $49,44 \pm 5,33^{* * *}$ & $55,46 \pm 1,20 * * *$ \\
\hline $120-180 \mathrm{Min}$. & $32,25 \pm 3,60$ & $31,50 \pm 2,83$ & $51,74 \pm 8,08 * * *$ & $52,47 \pm 4,73^{* * *}$ & $58,74 \pm 1,12 * * *$ \\
\hline $180-240 \mathrm{Min}$. & $33,40 \pm 3,45$ & $32,63 \pm 2,90$ & $53,65 \pm 8,03^{* * *}$ & $54,20 \pm 4,77 * * *$ & $60,38 \pm 1,50 * * *$ \\
\hline 240-300 Min. & $34,33 \pm 3,38$ & $33,48 \pm 2,95$ & $54,69 \pm 8,28 * * *$ & $55,31 \pm 4,94 * * *$ & $61,71 \pm 2,00 * * *$ \\
\hline
\end{tabular}


stündigen ${ }^{14} \mathrm{C}$-Einbau ins Plasma- bzw. Leberprotein irgendwelche statistisch signifikante Veränderungen gegenüber den Kontrollversuchen festgestellt werden. 6, 10 und 15 Tage nach der Verbrennung treten im Vergleich zu den Kontrollen signifikante Unterschiede auf. Die ${ }^{14} \mathrm{CO}_{2}$-Abatmung ist dabei signifikant erhöht. Am 6. Tage nach der Verbrennung beträgt die abgeatmete Dosis am Ende der fünfstündigen Versuchszeit $54,7 \%$, am 10 . Tage $55,3 \%$ und am 15 . Tage $61,7 \%$ gegenüber 34,3\% der Kontrollversuche.

Gleichzeitig ist der ${ }^{14} \mathrm{C}$-Einbau ins Leberprotein signifikant vermindert. Der ${ }^{14} \mathrm{C}$-Einbau bei den Kontrolltieren betrug $150530 \mathrm{Imp}$./Min. - g Protein. Er ist am 6. Tage auf $117568 \mathrm{Imp}$./Min. - g Protein, am 10. Tage auf $103465 \mathrm{Imp}$./Min. · g Protein und am 15. Tage auf 105413 Imp./Min. 'g Protein abgefallen. Der Einbau in die Plasmaproteine war dagegen bis zum 15. Tage nach der Verbrennung nicht signifikant verändert.

Einfluß von Verbrennungen auf den Leucinstoffwechsel der isoliert perfundierten Rattenleber

Auch an isoliert perfundierten Rattenlebern untersuchten wir den Einflu $ß$ von Verbrennungen auf den Leucinstoffwechsel. Wir bestimmten wiederum nach Leucin-[1-14 $\mathrm{C}]$-Gabe den Prozentsatz, der als ${ }^{14} \mathrm{CO}_{2}$ freigesetzt wurde, und den, der ins Leber- und Plasmaprotein eingebaut wurde (Abb. 2).

Die Werte bezogen wir auf $\mathrm{g}$ Leber, da anzunehmen ist, $\mathrm{da} ß$ eine größere Leber auch einen entsprechend

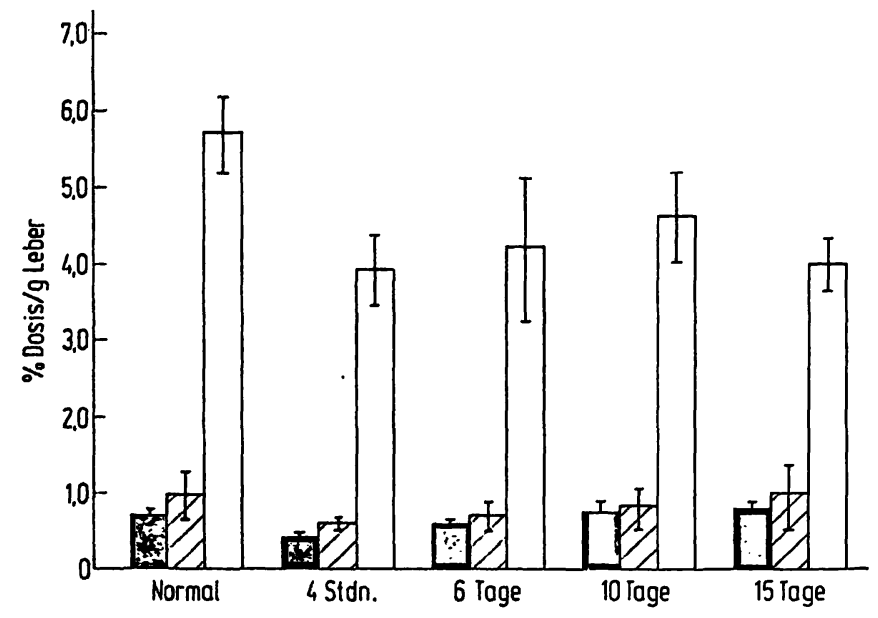

Abb. 2

"CO $\mathrm{CO}_{2}$ Abatmung (schwarze Säulen) und Leucin-[1-"'C]-Einbau in die Plasmaproteine (schraffierte Säulen) und das Leberprotein (weiße Säulen) isoliert perfundierter Rattenlebern von normalen und verbrannten Ratten. Auf der Abszisse sind die Zeiten nach Verbrennung angegeben. Die Messung der eingebauten bzw. abgeatmeten "C-Aktivität erfolgte 1 Std. nach Zugabe von $2 \mu \mathrm{C}$ Leucin-[1-14C] zum Perfusionsmedium. "C-Aktivitäten sind auf das Lebergewicht bezogen und angegeben als Prozentsatz der zugeführten "'C-AKtivität jeweils als Mittelwert von mindestens 4 Einzelwerten \pm Standardabweichung

höheren Stoffwechsel besitzt (vgl. Tab. 4). Der Versuch bei den Normaltieren zeigte, daß die über 2 Stdn. abgeatmete ${ }^{14} \mathrm{CO}_{2}$-Menge ungefähr auf dem gleichen Niveau blieb. Der Einbau ins Leberprotein dagegen erfolgte ziemlich schnell. Nach 30 Min. Perfusionsdauer sind schon 4,92\% Dosis/g Leber eingebaut worden. In den nächsten $1 \frac{1}{2}$ Stdn. kamen nur noch

Tab. 4

${ }^{1 *} \mathrm{CO}_{2}$-Abatmung $(\overline{\mathrm{x}} \pm \mathrm{s})$ isoliert perfundierter Rattenlebern nach Zugabe von Leucin-[1-1*C], $2 \mu \mathrm{C} / 60 \mathrm{ml}$ Perfusionsmedium. Die Lebern stammten von Ratten, die 4 Stdn., 6, 10 oder 15 Tage vor der Organentnahme eine standardisierte Verbrennung erhielten sowie von unverbrannten Kon$* p<0,05 \quad * * p<0,01 \quad * * p<0,001$

\begin{tabular}{lcccc}
\hline $\begin{array}{l}\text { Zeit nach } \\
\text { Leucin- } \\
\text { injektion }\end{array}$ & Kontrollen & 4 Stunden & 6 Tage & 10 Tage \\
& $\cdot n=5$ & $n=4$ & $n=4$ & $n=4$ \\
\hline
\end{tabular}

\section{$0-30 \mathrm{Min}$.}

30- $60 \mathrm{Min}$.

90-120 Min.

0 - $30 \mathrm{Min}$.

30- $60 \mathrm{Min}$.

90-120 Min.

$0,706 \pm 0,064$

$0,778 \pm 0,064$

$0,492 \pm 0,044$

${ }^{1} \mathrm{COO}_{2}$ ( $\%$ der Dosis/Std. - $\mathrm{g}$ Leber)

$\begin{array}{ll}0,398 \pm 0,098 * * & 0,508 \pm 0,092 * \\ 0,456 \pm 0,048 * * * & 0,644 \pm 0,034 \\ 0,320 \pm 0,052 & 0,450 \pm 0,071 \\ 0,332 \pm 0,048 * & 0,646 \pm 0,130\end{array}$

$0,320 \pm 0,052$

$0,646 \pm 0,130$

${ }^{14} \mathrm{CO}_{3}$ (\% der Dosis/Std. $\cdot \mathrm{g}$ Leber (kumulativ))

$0,200 \pm 0,049 * * \quad 0,253 \pm 0,054 * *$

$0,200 \pm 0,049 * *$
$0,428 \pm 0,077 * *$

$0,588 \pm 0,095 * *$
$0,754 \pm 0,087 * *$

$0,253 \pm 0,054 * *$
$0,575 \pm 0,071 * *$
$0,800 \pm 0,085 *$

$0,800 \pm 0,085$
$1,123 \pm 0,151$

$\begin{array}{lll}0,353 \pm 0,032 & 0,722 \pm 0,051 & 0,57 \pm \pm 0,07 * * \\ 0,968 \pm 0,069 & 0,588 \pm 0,095 * * & 0,800 \pm 0,085 * \\ 0,250 \pm 0,092 & 0,754 \pm 0,087 * & 1,123 \pm 0,151\end{array}$

Tab. 5

Einbau von Leucin-[1-14C] $(\overline{\mathbf{x}} \pm s)$ isoliert perfundierter Rattenlebern in Leber- und Plasmaproteine. Die Lebern stammten von Ratten, die 4 Stdn., 6, 10 oder 15 Tage vor der Organentnahme eine standardisierte Verbrennung erhielten sowie von unverbrannten Kontrolltieren. Leucindosis $2 \mu \mathrm{C} / 60 \mathrm{ml}$ Perfusionsmedium.

\begin{tabular}{lccccc} 
& & $* \mathrm{p}<0,05$ & $* * \mathrm{p}<0,01$ & $* * \mathrm{p}<0,001$ & \\
\hline $\begin{array}{l}\text { Zeit nach } \\
\text { Leucin- } \\
\text { injektion }\end{array}$ & Kontrollen & 4 Stunden & 6 Tage & 10 Tage \\
\hline
\end{tabular}

\begin{tabular}{|c|c|c|c|c|c|}
\hline \multicolumn{6}{|c|}{ Leucineinbau in Leberprotein [\% Dosis/g Leber] } \\
\hline $\begin{array}{l}30 \text { Min. } \\
60 \text { Min. } \\
120 \text { Min. }\end{array}$ & $\begin{array}{l}4,92 \pm 1,02 \\
5,71 \pm 0,54 \\
6,69 \pm 1,37\end{array}$ & $\begin{array}{l}3,14 \pm 0,35 * * \\
3,92 \pm 0,44 * * \\
4,95 \pm 0,61 *\end{array}$ & $\begin{array}{l}3,22 \pm 0,55^{*} \\
4,40 \pm 0,96 * \\
5,38 \pm 1,10\end{array}$ & $\begin{array}{l}3,36 \pm 0,49 * \\
4,59 \pm 0,61^{* *} \\
5,77 \pm 0,57\end{array}$ & $\begin{array}{l}3,07 \pm 0,24 * * \\
3,95 \pm 0,33^{* * *} \\
4,79 \pm 0,066^{*}\end{array}$ \\
\hline $\begin{array}{l}30 \text { Min. } \\
60 \text { Min. } \\
120 \text { Min. }\end{array}$ & $\begin{array}{l}0,252 \pm 0,11 \\
0,972 \pm 0,287 \\
1,748 \pm 0,422\end{array}$ & $\begin{array}{l}0,122 \pm 0,04^{*} \\
0,600 \pm 0,077^{*} \\
1,435 \pm 0,249\end{array}$ & $\begin{array}{l}0,156 \pm 0,026 \\
0,684 \pm 0,21 \\
1,260 \pm 0,400\end{array}$ & $\begin{array}{l}0,162 \pm 0,017 \\
0,791 \pm 0,255 \\
1,735 \pm 0,446\end{array}$ & $\begin{array}{l}0,292 \pm 0,115 \\
0,970 \pm 0,43 \\
1,765 \pm 0,67\end{array}$ \\
\hline
\end{tabular}


1,77\% Dosis/g Leber hinzu. Anders sah es beim Einbau ins Plasmaprotein aus. Wir stellten hier eine Verzögerung fest. Nach $30 \mathrm{Min}$. wurden erst 0,252\% Dosis/g Leber gemessen, nach 60 Min. dann 0,972\% Dosis/g Leber und nach 120 Min. 1,748\% Dosis/g Leber.

Die Lebern aller verbrennungskranken Tiere bilden in den ersten 30 Min. der Perfusionsversuche signifikant weniger ${ }^{14} \mathrm{CO}_{2}$ aus der eingegebenen ${ }^{14} \mathrm{C}$-Leucinmenge. Nach Ablauf von 60 Min. konnten nur noch die Lebern von $4 \mathrm{Stdn}$. und 6 Tage vorher verbrannten Ratten signifikant weniger ${ }^{14} \mathrm{CO}_{2}$ freisetzen. Die Werte von den 10 Tage und 15 Tage vorher verbrannten Tieren überschritten bereits nach $60 \mathrm{Min}$. langer Perfusion die Normalwerte. Die Unterschiede waren aber statistisch nicht zu sichern.

Das Verhalten der ${ }^{14} \mathrm{CO}_{2}$-Freisetzung änderte sich im Prinzip auch nach einer Perfusionszeit von 90 und 120 Min. nicht mehr. Dabei lagen die 4 Stdn.- und 6-Tage-Werte signifikant unter dem Normalniveau, während die 10-Tage- und 15-Tage-Werte die Norm nicht signifikant überschritten (Tab. 4).

Der ${ }^{14} \mathrm{C}$-Einbau in die Plasmaproteine ist $4 \mathrm{Stdn}$. nach der Verbrennung über den ganzen. Perfusionsversuch (30-120 Min.) signifikant vermindert. Die 6-TageWerte sind ebenfalls vermindert; sie liegen aber nur an der Grenze der Signifikanz. Die 10-Tage-Werte unterscheiden sich statistisch nicht mehr von denen der Kontrolliversuche. 15 Tage nach der Verbrennung konnten größere ${ }^{14} \mathrm{C}$-Einbauraten in die Plasmaproteine gemessen werden. Die Mittelwerte konnten aber statistisch nicht gesichert werden (Tab. 5).

Ähnlich wie bei den in vivo Versuchen wurde bei fast allen Lebern von verbrannten Tieren zu den Kontrollzeiten (30,60 und $120 \mathrm{Min}$.) signifikant vermindert ${ }^{14} \mathrm{C}$ in das säurefällbare Leberprotein eingebaut. Die Werte nach 2 stdg. Perfusion konnten 6 und 10 Tage nach der Verbrennung nicht statistisch gesichert werden.

\section{Diskussion}

In Übereinstimmung mit früheren Untersuchungen (1) finden wir eine Zunahme der Summe der freien Aminosäuren im Plasma nach Verbrennung. Dabei steigt der Aminosäuregehalt zunächst in der Schockphase steil an, fällt danach ab und steigt dann erneut in der späten Phase der Verbrennung an. Auch das hatten wir früher so beobachtet. Die Unterschiede in den Absolutwerten dürften im wesentlichen dadurch bedingt sein, daß in der früheren Untersuchung (1) Leucinäquivalente zugrunde gelegt wurden, während jetzt die einzelnen Aminosäuren bestimmt und die Einzelwerte addiert wurden.

Die Kinetik der einzelnen Aminosäurespiegel folgt nicht immer der Kinetik der Gesamtaminosäuren im Plasma. Manche Aminosäuren sind zeitweise im Plasma signifikant abgefallen, z. B. Threonin, Serin, Glycin, Methionin, während die Summe der Aminosäuren höher als normal ist. In der Schockphase sind es vor allem die nicht zu den Aminosäuren zählenden Substanzen Taurin und Harnstoff, die den Anstieg des „säurelöslichen $\alpha$-Aminostickstoffs“ bedingen. In der Spätphase geht der Anstieg der Gesamtaminosäuren auf das Konto einiger Aminosäuren, während andere durchaus im Normbereich sind.

Die unterschiedlichen Muster der Plasmaaminosäuren in der Schockphase und in der Spätphase der Verbrennung werten wir als Ausdruck der unterschiedlichen Ursachen für die frühen und die späten Veränderungen nach Verbrennung'. Eine Bestätigung dafür bringen die Ergebnisse der Leucinstoffwechseluntersuchungen. Wenige Stunden nach der Verbrennung ist weder der Einbau von Leucin in Leberund Plasmaproteine, noch der Abbau von Leucin, gemessen als ${ }^{14} \mathrm{CO}_{2}$-Bildung, signifikant verändert. Ein und zwei Wochen nach der Verbrennung besteht dagegen ein deutlicher Katabolismus mit einem verminderten Einbau in die Leberproteine und einem fast auf das Doppelte gesteigerten Leucinabbau. Es sind also nicht nur die großen Stickstoffverluste durch das lokal bei der Verbrennung zerstörte Protein, die die Stickstoffbilanz negativ gestalten (2). Wenn aber zugeführte Aminosäuren wie z. B. Leucin vom verbrannten Organismus im gesteigerten Maße abgebaut werden, muß auch der therapeutische Nutzeffekt einer erhöhten Eiweißzufuhr (15) zweifelhaft bleiben.

Erstaunlich ist, daß nur der Einbau von Leucin in das Leberprotein gestört ist, nicht aber in die Plasmaproteine, die auch zu einem Großteil in der Leber synthetisiert werden. Dieser Befund wird bestätigt durch die Ergebnisse an der isoliert perfundierten Leber. Hier sind ebenfalls nur die Leberproteine durch die Synthesehemmung betroffen (Tab. 5). Auch wird von der isolierten Leber verbrannter Ratten nicht wie am Ganztier vermehrt Leucin $\mathrm{zu}^{14} \mathrm{CO}_{2}$ abgebaut, obwohl doch gerade die Leber das Hauptorgan für den Aminosäurestoffwechsel ist.

Es müssen also mindestens zwei Effekte sein, die bei der Störung des Proteinstoffwechsels in der späten Phase der Verbrennungskrankheit zusammenwirken. Der eine ist gekennzeichnet durch den stark erhöhten Abbau von Leucin zu $\mathrm{CO}_{2}$. Er ist abhängig von der Körperperipherie. $\mathrm{Ob}$ es die veränderte Aminosäurezusammensetzung ist (16), sollen weitere Untersuchungen klären. $\mathrm{Zu}$ denken ist in diesem Zusammenhang auch an einen zusätzlichen Energieverlust durch Verdunstungswärme $(17,18,19)$.

Unabhängig von diesen Effekten, die nur am Ganztier beobachtet werden können, ist jedoch die Proteinsynthese der isolierten Leber gestört. Es muß ein sehr spezifischer Effekt sein, der zu dieser Hemmung der Synthese von Leberproteinen führt, denn die Plasmaproteinsynthese dieser Lebern ist nicht gehemmt (Tab. 5). Die früher von uns beobachtete Energiestoffwechselstörung (1) reicht also zur vollständigen Erklärung dieser Phänomene nicht aus.

Herrn Dr. K.-H. RudoRfF danken wir für die Durchführung der Leberperfusion.

Mit Unterstützung der Europäischen Gemeinschaften durchgeführte Forschungsarbeit. 


\section{Literatur}

1. KrönER, H., E. MEINKEN und W. Statb, diese Z. 5, 81 (1967).2. AlIGöwer, M., J. SiEgrist, Verbrennungen, Springer-Verlag Berlin-Heidelberg-New York (1957). - 3. RüENAUVER, R., H. KRöNER und W. Staib, Hoppe-Seyler's Z. physiol. Chem. 336, 227 (1964). - 4. MOORE, S. und W. H. Stern, J. biol. Chemistry 211, 915 (1954). - 5. Kleninhaus, H., H. Franz und H. ZimmermannTelschow, Z. analyt. Chem. 214, 175 (1965). - 6. KonnenSснмӥhling, R., Dissertation, Univ. Düsseldorf (1966). - 7. Jefrray, H. und J. Alvarez, Analytic. Chem. 33, 612 (1961). 8. Herberg, R. J., Analytic. Chem. 32, 42 (1960). - 9. Weichselbaum, T. E., Amer. J. Clin. Path. 16, 40 (1946). - 10. Meiers, H. G., G. FlammanN und W. Staib, Biochem. Z. 344, 514 (1966). 11. K. H. RudORFF, Dissertation, Univ. Düsseldorf (1966). -
12 Schrmassex, $H$., in: Stoffwechsel der isoliert perfundierten Leber, Hrsg. W. Staib und R. Scholz, Springer-Verlag, BerlinHeidelberg-New York (1968). - 13. MiLLER, L. L., C. G. BLY, M. L. Watson und W. F. Bale, J. Exper. Med. 94, 431 (1951). 14. Exton, J. H., C. R. PArk, J. biol. Chemistry 242, 2622 (1967).15. Müller, F. E., Med. Klin. 60, 636 (1965). - 16. KNAUfF, H. G., G. Mayer, W. Scholl und B. Miller, Dtsch. med. Wschr. 94, 1057 (1969). - 17. Harrison, H. N., J. A. Moncrief, J. W. Ducketr und A. D. Mason, Surgery (St. Louis) 56, 203 (1964). 18. Horn, L., J. M. Converse, Amer. J. Physiol. 207, 861 (1964).19. Caldewell, F. T. und K. Levitsky, Arch. Surg. (Chicago), 86,500 (1963).
Priv.-Doz. Dr. H. Kröner Inst. f. Physiol. Chemie 4000 Düsseldorf Witzelstr. 111 